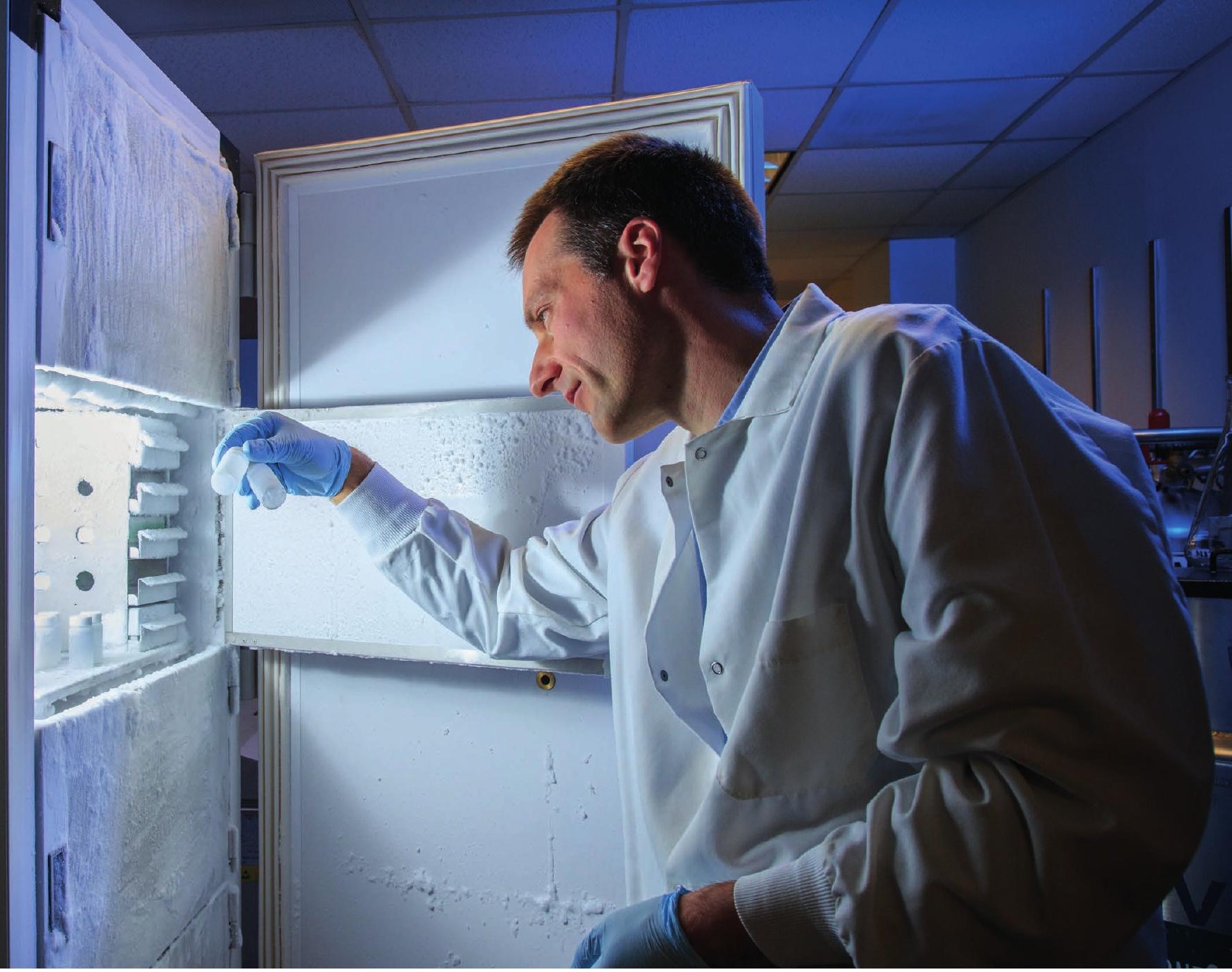

Matthias Stephan aims to create off-the-shelf injectable nanoparticles that boost the body's ability to fight tumours.

T-CELL ENGINEERING

\title{
Attack of the killer clones
}

\section{The next generation of modified T-cell therapies is taking on solid tumours - but it's an uphill fight.}

\section{BY MEGAN SCUDELLARI}

$\mathrm{M}$ ost oncologists only dream of results this good. For the 63 children with B-cell acute lymphoblastic leukaemia (ALL) and no remaining treatment options, the chances of survival were slim. But no fewer than $83 \%$ achieved complete remission within three months of a single treatment with an experimental drug.

The drug, developed in the lab of Carl June at the University of Pennsylvania in Philadelphia, and advanced into clinical trials by pharmaceutical giant Novartis, is an engineered cell therapy. The patient's T cells (a type of white blood cell) are isolated from a blood sample and a genetic sequence encoding a chimaeric antigen receptor (CAR) protein is introduced into them. Once expressed, the CAR proteins prime the T cells to hunt down and kill cancer cells that present the antigen corresponding to that receptor. These CAR T cells, which June refers to as "serial killer" cells (because a single CAR T cell can destroy thousands of cancer cells), seemed to be very good at their job.

Ezra Cohen, co-director of the San Diego Center for Precision Immunotherapy at the University of California, San Diego, recalls sitting in the audience listening to June describe the results of the trial. A team engineered the children's T cells to home in on a protein called CD19, found on the surface of B cells, another type of white blood cell. "Just as CD19 is a nice target for leukaemias - but obviously doesn't exist on solid tumours - I thought there must be a counterpart or counterparts on solid malignancies," says Cohen. "That's when I began to get interested."

He wasn't the only one. Today, dozens of research programmes, both in academia and industry, are seeking to use the CAR-T-cell platform (CAR-T) in a wide range of cancers. CAR-T therapies have given good results against several types of blood cancer, including the most common type of lymphoma. And in August 2017, the US Food and Drug Administration (FDA) green-lit the Novartis anti-CD19 treatment (known as tisagenlecleucel) for B-cell ALL, the world's first engineered T-cell therapy to be approved.

The leap to solid tumours was inevitable - but not guaranteed to succeed. It's a bit like the Wild West right now, says Michael Brown, head of the translational oncology laboratory at the University of South Australia in Adelaide. Companies are pouring money into the field, trying to get a foothold, but initial attempts to deploy CAR-T therapies in solid tumours have had little success in reducing tumour size. "I'm hopeful, but 
it's obvious that the other results would need to parallel those in haematological malignancies, and that has not been evident," says Brown.

To make CAR-T work in solid tumours, researchers must improve the safety of the therapy, which has poorly understood - and sometimes deadly - side effects. They must also find ways to get the $T$ cells to penetrate the physical and immune fortress of a solid malignant mass. And scientists will need to come up with cheaper, faster ways to produce these therapeutic cells. Currently, it takes weeks to produce a single small batch of CAR T cells using specialized processing facilities.

"We're clearly in early stages, but I really do believe that CAR T cells will be an effective therapeutic strategy even in solid tumours, where there's a lot of scepticism right now," says Cohen.

\section{LESSONS LEARNT}

In July, when an FDA advisory committee voted unanimously to recommend approval of the Novartis anti-CD19 therapy, one committee member said it was the most exciting thing he had seen in his life.

The vote and the subsequent FDA approval are "a public validation" of the technology for these paediatric cancers, says David Chang, chief medical officer at Kite Pharma, a Santa Monica, California-based subsidiary of Gilead Sciences that has several CAR-T therapies in development. In October, Kite received FDA approval for its lead candidate, axicabtagene ciloleucel, for patients with relapsed or refractory large B-cell lymphoma. Other drug developers are likely to follow this path.

But not all CAR-T clinical trials have gone as well as the Novartis therapy. In March, Juno Therapeutics, based in Seattle, Washington, halted development of its leading CAR-T treatment after five patients in a phase II trial died following cerebral oedema (see page S74). This type of severe brain swelling is a major problem plaguing CAR-T therapies, and researchers are not yet sure of its precise cause.

Nor is it the only potentially dangerous side effect of CAR-T treatment. Infusing hyperactive immune cells into the body can have extreme consequences. During Novartis's 2015 trial, almost half of the participants had a severe inflammatory reaction called cytokine release syndrome, or a 'cytokine storm', now known to be a common side effect of CAR-T therapy. In a recent phase I trial for multiple myeloma in China, $85 \%$ of the first 35 patients experienced cytokine storms, although the effects can be controlled by medications ${ }^{1}$.

Because CAR-T therapies are so new, little is known about the long-term effects of treatment. One precautionary measure is to engineer a 'suicide' switch into the cells that is activated by a small-molecule compound, giving physicians the ability to promptly destroy the cells when needed - for example, in cases of brain swelling. Other approaches, including one being explored by Kite, use small molecules as switches to either increase or diminish T-cell activity by switching on or off their CAR proteins. Such control mechanisms seem poised to become common in secondand third-generation CAR-T therapies.

As the field looks to solid tumours, perhaps the most salient safety factor will be choosing the correct target antigens. Pick an antigen that is too rare, and you will not eradicate all the cancer cells. Pick an antigen that's ubiquitous, and you will also kill non-cancerous cells, imperilling the patient's health.

One reason that leukaemia and lymphoma have proved so susceptible to CAR-T treatments is that the target protein CD19 is expressed on all tumour cells in those cancers. Unfortunately, says Chang, "finding the right target in solid tumours has been a little bit more difficult than haematological malignancies.'

\section{SOLID TARGETS}

CAR-T researchers point to a single case that gives them hope that such targets exist. A 50-year-old man with a brain tumour that had spread to his spine, and who failed to respond to every other treatment, recovered following multiple doses of a CAR-T therapy infused directly into his central nervous system. The $T$ cells were engineered to attack tumour cells expressing an IL-13 receptor protein that is overexpressed in most cases of this type of brain tumour, called glioblastoma.

The patient was still in remission seven and a half months after the infusions, when his doctors at City of Hope Cancer Center in Duarte, California, published the results, the first of a phase I trial' ${ }^{2}$.

Other solid-tumour targets are being put to the test. In 2013, Brown initiated a clinical trial targeting CAR T cells to a protein called GD2 on the surface of metastatic melanoma cells. The trial, conducted in collaboration with Baylor College of Medicine in Houston, Texas, has treated six patients so far. Although the treatment seems to be safe, its efficacy remains in doubt. The CAR $\mathrm{T}$ cells have struggled to thrive and multiply

\section{"We have to come up with an idea that is easy to apply, easy to manufacture, and is as affordable as chemo- therapy."} inside the body, says

Brown. In only one of the six patients did the engineered $\mathrm{T}$ cells persist beyond four months ${ }^{3}$.

At the University of California, San Diego, Cohen, along with oncologist Thomas Kipps and colleagues, is focusing on a receptor protein called ROR1 that is expressed in many aggressive, difficult-to-treat cancers. The team has shown that CAR T cells engineered to recognize ROR1 preferentially kill ROR1expressing cancer cells in preclinical models of head and neck, pancreatic, lung, and a type of breast cancer known as triple-negative (due to the absence of receptors for three specific hormones). Cohen and Kipps plan to move into early-phase clinical trials by the end of 2018. Juno Therapeutics also has an anti-ROR1 CAR-T therapy in development, already in a phase I clinical trial.

At the Memorial Sloan Kettering Cancer Center in New York City, scientists are targeting a protein called mesothelin, which is overexpressed in lung, ovarian, breast and pancreatic cancers. Similar to ROR1, mesothelin is associated with worse outcomes and tumour metastasis.

Prasad Adusumilli, a thoracic surgeon and researcher at Sloan Kettering, conducted a 2014 study, along with immunologist Michel Sadelain, which showed that mesothelintargeting CAR T cells directly administered into the chest cavity of mice multiplied and could wipe out tumours ${ }^{4}$. Adusumilli's team now has two phase I clinical trials of these $\mathrm{T}$ cells in progress. So far, they have treated nine patients in a trial for activity against mesothelioma, lung or breast cancer, and five more in a trial for triple-negative breast cancer.

Yet even with the right targets, it remains an open question whether CAR-T therapies will ever work as well for solid tumours as they do for blood cancers.

To beat a solid tumour, an engineered cell therapy must do four things: get to the tumour, penetrate the tumour, battle an immunosuppressive environment, and kill the target cancer cells. The first and last steps seem to be the easiest. It is steps two and three that stymie current efforts.

Take, for example, a recent glioblastoma trial at the University of Pennsylvania. The study showed that a CAR-T therapy targeting the EGF receptor III protein, delivered by an injection into the hand, did reach and penetrate brain tumours, killing tumour cells. But within two weeks of the injection, the number of T cells went into decline. "What was most unexpected was a very strong, adaptive, compensatory immunosuppression," says senior author Marcela Maus, now at Massachusetts General Hospital in Boston.

To counter the formidable ability of solid tumours to suppress the immune system around them, many researchers, including Cohen, Brown and Adusumilli, are testing their therapies in conjunction with drugs known as checkpoint inhibitors. These are immune-modulating agents that block the ability of tumours to shield themselves from the body's immune system.

To be effective against solid tumours, CAR T cells will probably need to be used in combination with other therapies. "People have the impression that these modified T cells 
would somehow be autonomous in action," says Brown. "But they're not." Rather, he explains, the cells are subject to the same difficulties in penetrating tumours and overcoming immunosuppressive environments as other therapies.

\section{REIMAGINING CAR-T}

Beyond the daunting biological hurdles, CAR-T therapies face time and cost issues. Chemotherapy is ubiquitous in oncology not only because it can slow or stop cancers, but because the drugs involved can be generated quickly and inexpensively. They can also be produced in relatively large amounts and easily shipped and stored.

CAR-T therapies are the opposite. Typically, the production of these cells requires the removal of a patient's immune cells from a blood sample at a hospital, freezing the cells, shipping them to a CAR-T factory, thawing them, their genetic engineering with a viral vector, freezing them for the return trip, rethawing, and finally infusing them back into the patient. For the Novartis therapy, this is estimated to take 22 days. Given the complexity and urgency of the process, the company has priced its anti-CD19 treatment at US $\$ 475,000$ for a single infusion - roughly seven times as much as a typical chemotherapy regimen. And that price tag for the therapy doesn't include the high cost of hospital stays and follow-up visits.

"If we really want to outcompete chemotherapy as a front-line therapy, we have to come up with an idea that is easy to apply, easy to manufacture, and is as affordable as chemotherapy," says Matthias Stephan, an immunologist at the Fred Hutchinson Cancer Research Center in Seattle.

One such idea is the development of 'allogeneic' CAR T cells - T cells derived from individuals other than the patient. These 'universal' off-the-shelf CAR T cells are already being used in young patients in the United Kingdom. Infants and small children are often too little to extract enough healthy $\mathrm{T}$ cells for conventional CAR- T therapy, so immunologist Waseem Qasim and colleagues at University College London took T cells from adult donors. They then genetically altered these T cells to disrupt two cell-surface molecules so that the cells could be used without being tissue-matched to the patient. In two babies with B-cell ALL, these universal T cells cleared the leukaemia and allowed the infants to undergo successful stem-cell transplants ${ }^{6}$.

Qasim hopes that allogeneic CAR T cells will eventually be not just a bridge to transplantation for children, but a route to a cure. Given the advances in CRISPR gene-editing technology, "we can probably target almost any gene at will in cells," he says.

Yet allogeneic cells can cause the same side effects as CAR T cells made from the patient's own cells. A trial of an allogeneic CAR-T therapy, sponsored by Paris-based gene-editing company Cellectis and targeted against a rare form of leukaemia, was halted in August when a patient died from the effects of a cytokine storm (S74).

An off-the-shelf allogeneic product could be made even cheaper with additional tweaking to eliminate the need to combine CAR-T therapy with a checkpoint-inhibitor drug. Yangbing Zhao and a team from June's lab are genetically engineering CAR T cells to knock out checkpoint proteins such as PD-1 that tumour cells can exploit to shut down T cells. The team simultaneously knocked out four genes, including genes responsible for checkpoint molecules, in an attempt to create universal $\mathrm{T}$ cells ${ }^{7}$. This technique creates $\mathrm{T}$ cells that can resist being eliminated by the tumour microenvironment in the absence of checkpoint-inhibitor drugs ${ }^{8}$.

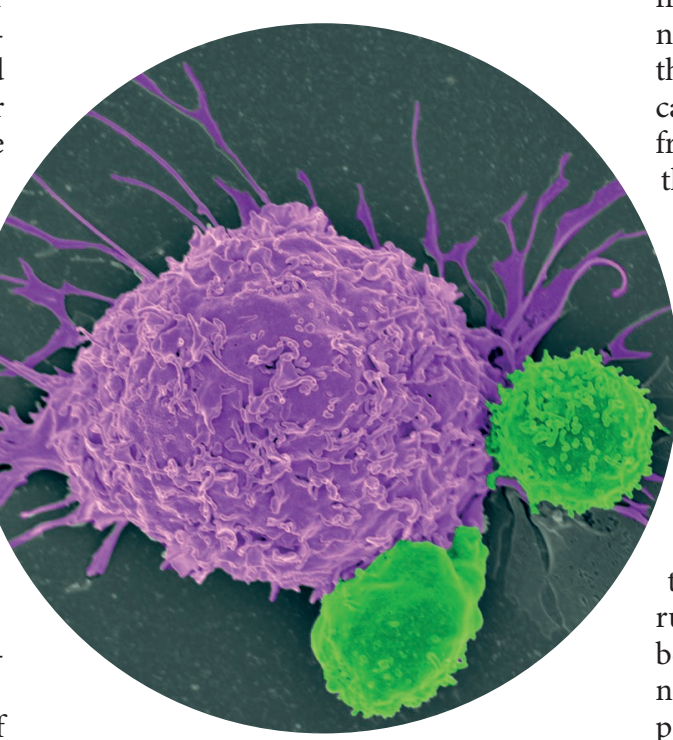

Pseudocolour image of chimaeric antigen receptor T cells attacking a tumour cell.

Last year, Adusumilli's team similarly added a PD-1 inhibitor to their CAR T cells by genetic engineering to improve outcomes in mouse models of lung cancer, and saw stronger antitumour effects and prolonged survival of the mice ${ }^{9}$.

In addition to gene editing, bioengineering approaches could slash the delivery and cost of engineered cell therapies, says Stephan. He and his colleagues at Fred Hutchinson think they can drop the CAR T-cell production time down to an afternoon - a speed-up of some 80 -fold - by eliminating most of the steps.

One technique involves programming T cells without ever removing them from the body. Biodegradable nanoparticles created in the lab carry DNA with genes encoding CAR proteins into the body. The nanoparticles stick like burrs to T cells and, once engulfed by the cells, deliver their genetic payload to the nucleus. In April, the team showed that these T-cell homing nanoparticles worked as well as infused CAR T cells in a mouse model of leukaemia, improving survival by 58 days on average compared with untreated mice ${ }^{10}$. Stephan expects that the cost of such a treatment will eventually be on a par with chemotherapy.

His team has also created CAR-T-cell-soaked patches for surgeons to apply directly to the site of a solid tumour. Using a sticky, spongy biopolymer that can be cut into various shapes as a scaffold, the researchers add a mix of growth-factor proteins to induce $T$ cells to survive and proliferate in the patch - just as they would in a human lymph node. "The challenge was to make a $3 \mathrm{D}$ environment where $\mathrm{T}$ cells believe they are in a lymph node, but they're sitting right on top of the tumour," says Stephan.

The scaffolds only require a relatively small number of T cells - around 4 million, compared with 1 billion for systemic infusion so it is not necessary to take so many $\mathrm{T}$ cells from patients or to spend days increasing their numbers in the lab, Stephan says. In tests of this technique on mouse models of pancreatic cancer and melanoma, CAR T cells migrated from the scaffolds into tumours and eradicated them more effectively than did the same cells introduced by systemic infusion ${ }^{11}$.

Even if creative delivery systems and genetic engineering lead to better CAR-T therapies for solid tumours, many researchers doubt whether they will ever reach the 80-90\% efficacy seen in blood cancers. However, such a therapy can claim an important space in oncology: although CAR-T treatments may not become the first choice for solid tumours, they could be used to treat patients who have run out of options, says Cohen. "I really do believe that we'll have a treatment that will be not only effective but even curative with some patients with highly refractory disease."

For this complicated and risky cell therapy, the most important move forward may be the continued effort to try new strategies in patients, pitting engineered cells against in situ tumour cells. "We don't know what makes the best CAR T cell until we put it into patients," says Maus. "It's just such a complex product."

Megan Scudellari is a freelance science reporter in Boston, Massachusetts.

1. Fan, F. et al. 2017 ASCO Ann. Meet. Abstr. LBA3001 (2017).

2. Brown, C. E. et al. N. Engl. J. Med. 375, 2561-2569 (2016).

3. Gargett, T. et al. Mol. Ther. 24, 1135-1149 (2016).

4. Adusumilli, P. S. et al. Sci. Transl. Med. 6, 261 ra151 (2014).

5. O’Rourke, D. M. et al. Sci. Transl. Med. 9, eaaa0984 (2017).

6. Qasim, W. et al. Sci. Transl. Med. 9, eaaj2013 (2017).

7. Ren, J. et al. Oncotarget 8, 17002-17011 (2017).

8. Ren, J. et al. Clin. Cancer Res. 23, 2255-2266 (2017).

9. Cherkassky, L. et al. J. Clin. Invest. 126, 3130-3144 (2016).

10.Smith, T. T. et al. Nature Nanotechnol. 12, 813-820 (2017).

11.Smith, T. T. et al. J. Clin. Invest. 127, 2176-2191 (2017). 